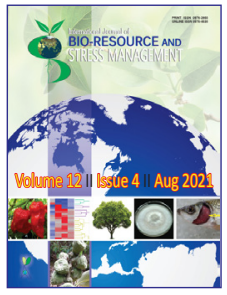

\title{
Deciphering the Molecular Architecture of a Candidate R-gene (BjuWRR1) Product Mediating White Rust Resistance in Brassica juncea
}

\author{
Chinmaya Kumar Das ${ }^{1 *}$, Umasankar Nayak ${ }^{2}$, Preetinanda Pati ${ }^{1}$, Mihir Ranjan Mohanty ${ }^{3}$, Sujata Das ${ }^{4}$, Parshuram \\ Sial $^{5}$, Bhagban Kabat ${ }^{2}$ and S. C. Swain 6
}

${ }^{1}$ AICRP on Rapeseed \& Mustard, Ranital, ${ }^{2}$ RRTTS, Ranital, ${ }^{3}$ RRTTSS, Jeypore, ${ }^{4}$ RRTTS, Keonjhar, ${ }^{5} \mathrm{HARS}$, Potangi, ${ }^{6} \mathrm{APC}$, Ranital, Odisha University of Agriculture \& Technology, Bhubaneswar, Odisha (756 111), India

Open Access

Corresponding Author

Chinmaya Kumar Das

e-mail: iitk.chinmaya@gmail.com

Citation: Das et al., 2021. Deciphering the Molecular Architecture of a Candidate R-gene (BjuWRR1) Product Mediating White Rust Resistance in Brassica juncea. International Journal of Bio-resource and Stress Management 2021, 12(4), 393-401. HTTPS://DOI. ORG/10.23910/1.2021.2205a.

Copyright: (c) 2021 Das et al. This is an open access article that permits unrestricted use, distribution and reproduction in any medium after the author(s) and source are credited.

Data Availability Statement: Legal restrictions are imposed on the public sharing of raw data. However, authors have full right to transfer or share the data in raw form upon request subject to either meeting the conditions of the original consents and the original research study. Further, access of data needs to meet whether the user complies with the ethical and legal obligations as data controllers to allow for secondary use of the data outside of the original study.

Conflict of interests: The authors have declared that no conflict of interest exists.

Acknowledgment: Authors are highly thankful for the contribution of CKD, UN, PP, MRM, SD, PS, BK and SS. CKD conceived the idea, did the bioinformatic analysis \& wrote the manuscript. CKD, UN \& PP help in the collection of literature. MRM, SD, PS, BK and SS help in the proofreading of the manuscript and formating of the manuscript.

\begin{abstract}
In this investigation, a three-dimensional model of a R-gene encoded product BjuWRR1 which is known to play a role in white rust resistance in Brassica juncea was developed to synthesize innovative ways for evolving white rust resistant cultivars. The model was built from the amino acid sequence of BjuWRR1 using structural template information of a disease resistance protein (RPP13-like protein 4 of Arabidopsis thaliana) with the help of homology-based modelling approach. Built models were validated for their stereochemical parameters and structural descriptors using Ramachandran plot analysis, protein structure analysis and ERRAT analysis. Structural analysis of BjuWRR1 model revealed that it is composed of three distinct domains namely a coiled-coil domain, a central NB-ARC nucleotide binding domain and a hypervariable leucine-rich repeat domain. Further, canonical conserved motifs such as P-loop, Kinase2-motif and HD-motif were found in the NB-ARC domain. The built model would help in understanding the molecular basis of plant-immunity against white rust pathogen by understanding the significance of inter-domain interactions in BuWRR1 in triggering the activation of downstream defense response against the white rust pathogen by promoting oligomerization of coiled-coil domains through stabilized hydrophobic interactions and interaction with NB-ARC domain. Presence of patches of charged residues in each domain of BjuWRR1 indicated their possible role in intra-molecular interaction with other domains. Therefore, this model can help in designing functional genomic studies to understand the role of intra-molecular interaction in BjuWRR1 to mediate resistance against white rust pathogen.
\end{abstract}

Keywords: Brassica juncea, resistance gene(R-gene), white rust

\section{Introduction}

Indian mustard (Brassica juncea (L.) Czern.) belonging to family brassicaceae is an important oilseed crop that plays a major role in shaping the edible oil economy of India by contributing $30 \%$ of its total edible oilseed production as well as the world by accounting for the second most source of vegetable oil and serving as the third most traded commodity across the globe (Anupriya et al., 2020; Kumar et al., 2009). White rust disease of Indian mustard caused by oomycete fungus Albugo candida (Pers.) Kuntze accounts for $60 \%$ yield loss in the globe (Saharan and Verma, 1992; Kamoun et al., 2015). Both "white pustules" (Heller and Thines, 2009) and "stag head" (Meena et al., 2014; Saharan et al.,

Article History

RECEIVED on $22^{\text {nd }}$ March 2021 RECEIVED in revised form on $14^{\text {th }}$ August 2021 ACCEPTED in final form on $30^{\text {th }}$ August 2021 
2014) are the characteristic symptoms seen in the host plant due to infection by white rust pathogen (Links et al., 2011). White rust pathogen cause significant yield losses in most of the commercially grown mustard cultivars in India. Therefore, development of genetic resistance against this pathogen can help in minimizing yield losses in Indian mustard (Kumar et al., 2021a; Kumar et al., 2021b). Both expressions of disease resistance genes or R-genes and loss or mutation in disease susceptibility genes or S-genes are involved in mediating genetic resistance (Anupriya et al., 2020). The majority of the plant disease resistance genes or R-genes encode for nucleotide-binding-site leucine rich repeat receptors or NLR proteins (Jones and Dangl, 2006; Maekawa et al., 2011). Plant NLR proteins play a direct or indirect role in immune sensing of typical isolate-specific pathogen effector proteins delivered into plant cells (Duxbury et al., 2016; Dodds and Rathjen, 2010). Activation of plant NLRs causes termination of pathogen proliferation by inducing an array of immune responses coupled with a form of localized cell death called hypersensitive response (HR) (Chisholm et al., 2006 ; Cui et al., 2015). NLR proteins are composed of a conserved tripartite domain structure with a nonconserved $\mathrm{N}$-terminal domain, a central nucleotide-binding (NB) domain, and a C-terminal LRR domain (Lukasik and Takken, 2009). Plant NLRs can be classified into three monophyletic classes based upon the variations in $\mathrm{N}$-terminal domains namely toll/interleukin1 receptor (TIR) NLRs (TNLs), Coiled-coil (CC) NLRs (CNLs) and NLRs containing an N terminal RPW8 domain (RNLs) (Shao et al., 2016). The central conserved NB region of plant NLRs is also known as the NB-ARC domain due to its presence in the founding members Apaf-1, resistance (R) proteins, and CED-4 (Jones and Dangl, 2006; Maekawa et al., 2011; Duxbury et al., 2016). The C-terminal LRR region of NLRs is highly variable (Takken and Goverse, 2012) which helps in the recognition of various effector structures of pathogen (Cui et al., 2015; Petit-Houdenot and Fudal, 2017).

In literature, most of the studies related to mapping of loci conferring resistance to white rust pathogen Albugo candida is reported in Arabidopsis thaliana (Borhan et al., 2001; Cevik et al., 2019) and in Brassica rapa (AA) (Kole et al., 1996; Kole et al., 2002), B. juncea (AABB) (Prabhu et al., 1998; PanjabiMassand et al., 2010) and Brassica napus (AACC) (Ferreira et al., 1995). Further, identification and cloning of two R-genes namely RAC1 and WRR4 in Arabidopsis thaliana is reported (Borhan et al., 2004, 2008). Similarly, a candidate gene BjuWRR1 encoding for a CC-NB-LRR protein conferring genetic resistance against white rust disease in Brassica juncea has been reported (Arora et al., 2019). Unfortunately, no three dimensional structural information on BjuWRR1 is available till date. In this investigation, molecular modeling based approach is attempted to construct the three-dimensional model of BjuWRR1 protein to understand the genetic basis of resistance at a molecular level.

\section{Materials and Methods}

\subsection{Sequence retrieval}

Amino acid sequence of a candidate R-gene BjuWRR1 encoding for a CC-NB-LRR type intracellular receptor in Brassica juncea [QEM22687.1] and its orthologues from other plant species in Brassica rapa [XP_033147307.1], Brassica napus [XP_013748433.1], Brassica oleracea [XP_013633813.1], Sinapsis alba[KAF8105614.1], Arabidopsis thaliana[NP_001319213.1], Arabidopsis lyrata [XP_002891747.1] and Capsella rubella [XP_023632900.1] were retrieved from the protein database of NCBI (https:// www.ncbi.nlm.nih.gov/protein) (Pruitt et al., 2005) for 3D model construction of BjuWRR1 and multiple sequence alignment.

\subsection{Molecular modelling}

Three dimensional model of BjuWRR1 was constructed using its amino acid sequence as "target" from the structural information of C-chain of disease resistance RPP13-like protein4 in Arabidopsis thaliana (6J5T.1.C.pdb). Molecular modelling was carried out using homology modelling based approach with the help of SWISS-Model (https://swissmodel. expasy.org/) (Waterhouse et al., 2018) webserver.

\subsection{Evaluation of models}

Built models were evaluated for their structural descriptors and stereochemical parameters, and only robust models were selected for further analysis. All the built models were subjected to protein structure analysis (ProSA analysis) and Ramachandran plot analysis for validation of the models.

\subsubsection{ProSA analysis}

Protein structure analysis was conducted using ProSA-web server (https://prosa.services.came.sbg.ac.at/prosa.php) (Wiederstein and Sippl, 2007 )to evaluate the accuracy of the built models by computing potential of $\mathrm{C} \alpha$ coordinates and energy of structures to produce Z-scores and residues energy plots. Z-score reflects the overall quality of the model while the energy plot of the model evaluates the local quality of the model (Sippl, 1993; Wiederstein and Sippl, 2007).

\subsubsection{Ramachandran plot analysis}

Accuracy of stereochemical parameters of built model was analyzed with the help of Ramachandran plot analysis using PROCHECK tools which inspects the accuracy of dihedral angles ( $\phi$ and $\psi$ ) for residue by residue geometry and overall structural geometry (Laskowski et al., 1993).

\subsubsection{ERRAT analysis}

ERRAT analysis plays a role in determining the overall quality of the model by examining the non-bonded atomic interactions. A higher ERRAT score is an indicator of a more native-like model (Colovos and Yeates, 1993).

\subsection{Visualization of the molecular architecture of model}

Molecular architecture of the built 3D-model of BjuWRR1 was visualized using UCSF Chimera (Pettersen et al., 2004) and with 
the help of an open-source graphic tool PyMol (DeLano, 2002).

\subsection{Multiple Sequence alignment and visualization}

Multiple sequence alignment of BjuWRR1 and its orthologues in different plant species was carried out using ClustalX (Jeanmougin et al., 1998) and were visualized using a multiple sequence alignment editor Jalview (Waterhouse et al., 2009).

\section{Results and Discussion}

Different stresses including abiotic and biotic stresses adversely affect the growth, development and yield performance of crop plants. Biotic stresses are primarily caused by plant pathogens and insect pests causing significant loss in crop production (Das et al., 2018a and 2018b; Das et al., 2021; Kumar et al., 2021a; Kumar et al., 2021b). Plants have evolved two different kinds of molecular circuits against invading pathogens namely PAMP (Pathogen associated molecular patterns)-triggered immunity (PTI) and effector-triggered immunity (ETI) in addition to physical and chemical barriers. PTI is considered as the first layer of defense response in plants in which conserved PAMPs of the invading pathogens are recognized using cell surface PRRs (Pattern recognition receptors). ETI is the second layer of defense response in plants in which pathogen secreted effectors are recognized by intracellular receptors mainly nucleotide-binding leucine rich receptors(NB-LRRs) encoded by resistance genes or R-genes (Jones and Dangl, 2006; Dodds and Rathjen, 2010). There are two different types of NB-LRR receptors namely TIR-NB-LRR (TNL) and CNL (CC-NB-LRR) depending upon the presence of a TIR (similar to animal Tolllike/interleukin-1 receptors) domain or CC (coiled-coil) domain in their N-terminal domain, respectively (Michelmore et al., 2013; Lee and Yeom, 2015). Recently, one candidate R-gene BjuWRR1 encoding for a CC-NB-LRR type intracellualr receptor conferring complete resistance against white rust disease caused by an oomycetes fungal pathogen Albugo candida in Brassica juncea line Donskaja-IV is reported (Arora et al., 2019). However, three dimensional structural information on BjuWRR1 protein is not available which is important for understanding the mechanism of action for genetic resistance mediated by such protein and also for developing functional haplotype-based markers to differentiate susceptible and resistant genotypes. In this study, an attempt has been made to decipher the molecular anatomy of BjuWRR1 protein using molecular modeling based approach using their amino acid sequence information and visualization of different conserved domains in the BjuWRR1 model using computational tools.

\subsection{Structural modeling of BjuWRR1}

Three-dimensional model of BjuWRR1 was constructed from its amino acid sequence information as "target" (QEM22687.1 WRR1) retrieved from the protein database of NCBI with the help of structural template information of C-chain of disease resistance RPP13-like protein4 in Arabidopsis thaliana (6J5T.1.C.pdb). The template had a $27.25 \%$ sequence identity and $33 \%$ sequence similarity with the target and covering $89 \%$ region of the target. The 3D-model of target BjuWRR1 using structural coordinates of the template was carried out using the SWISS-Modeller server (https://swissmodel.expasy.org/) (Waterhouse et al., 2018). The built 3D model of BjuWRR1 was evaluated for different stereochemical and structural quality parameters using a set of structural validation tools namely PROCHECK (Laskowski et al., 1993), ProSA (Sippl, 1993; Wiederstein and Sippl, 2007), and ERRAT (Colovos and Yeates, 1993). Such analysis reflected BjuWRR1 model had a sufficient score for accuracy and reliability. PROCHECK analysis of the BjuWRR1 model revealed the presence of $99 \%$ residues in the allowed region ( $81.5 \%$ in the most favoured core region, $16.2 \%$ in additional allowed region and $1.4 \%$ in generously allowed region) and only $0.9 \%$ residues in the disallowed region of Ramachandran plot indicating moderate reliability of stereochemical parameters (Figure 1d). Similarly, ERRAT analysis revealed an overall quality factor of 86.4 was obtained for BjUWRR1 indicating the model to be a more native like model. ProSA analysis of the BjuWRR1 model indicated that a z-score of -9.19 for overall quality is within the range of the native protein structures (Figure 1a). Further, the local quality of the BjuWRR1 model reflected a negative energy-rich region for the most region of the protein except few regions of the $\mathrm{C}$-terminal in the energy plot (Figure $1 \mathrm{~b}$ and $1 \mathrm{c}$ ). Therefore, protein structure analysis using ProSA confirmed the reliability of our model.

\subsection{Molecular anatomy of R-protein BjuWRR1}

BjuWRR1 is a candidate R-protein involved in mediating white rust resistance in Brassica juncea (Arora et al., 2019). In general, nucleotide-binding leucine-rich repeat(LRR) receptors (NLR) play a role in mediating plant-immunity by detecting the effector proteins of pathogens (Wang et al., 2019). In order to understand the molecular anatomy of BjuWRR1, we built the model of BjuWRR1 using homology-based modelling approach. The three dimensional model of BjuWRR1 was found to be composed of three distinct conserved canonical domains namely a coiled-coil domain, NB-ARC domain and an LRR domain which are typically found in nucleotidebinding leucine-rich repeat receptor proteins involved in the modulation of plant immunity. The details of each domain are described in the following sections.

\subsubsection{Coiled-coil domain}

Visualization of molecular anatomy of BjuWRR1 model revealed that it contains a coiled-coil (CC) domain in its $\mathrm{N}$-terminal which is typically found in different NLR type $\mathrm{R}$-protein members. $\mathrm{N}$-terminal coiled-coil domain is known to act as a likely signaling module in plant NLR which play a role in the induction of hypersensitive response (HR) induced cell death and disease resistance response (Swiderski et al., 2009; Collier et al., 2011; Maekawa et al., 2011; Bernoux et al., 2011 and Cesari et al., 2016). In BjuWRR1, the N-terminal coiled-coil region spans from Val5 residue to His133 residue consisting of three alpha-helices. CC-region is mainly rich in 

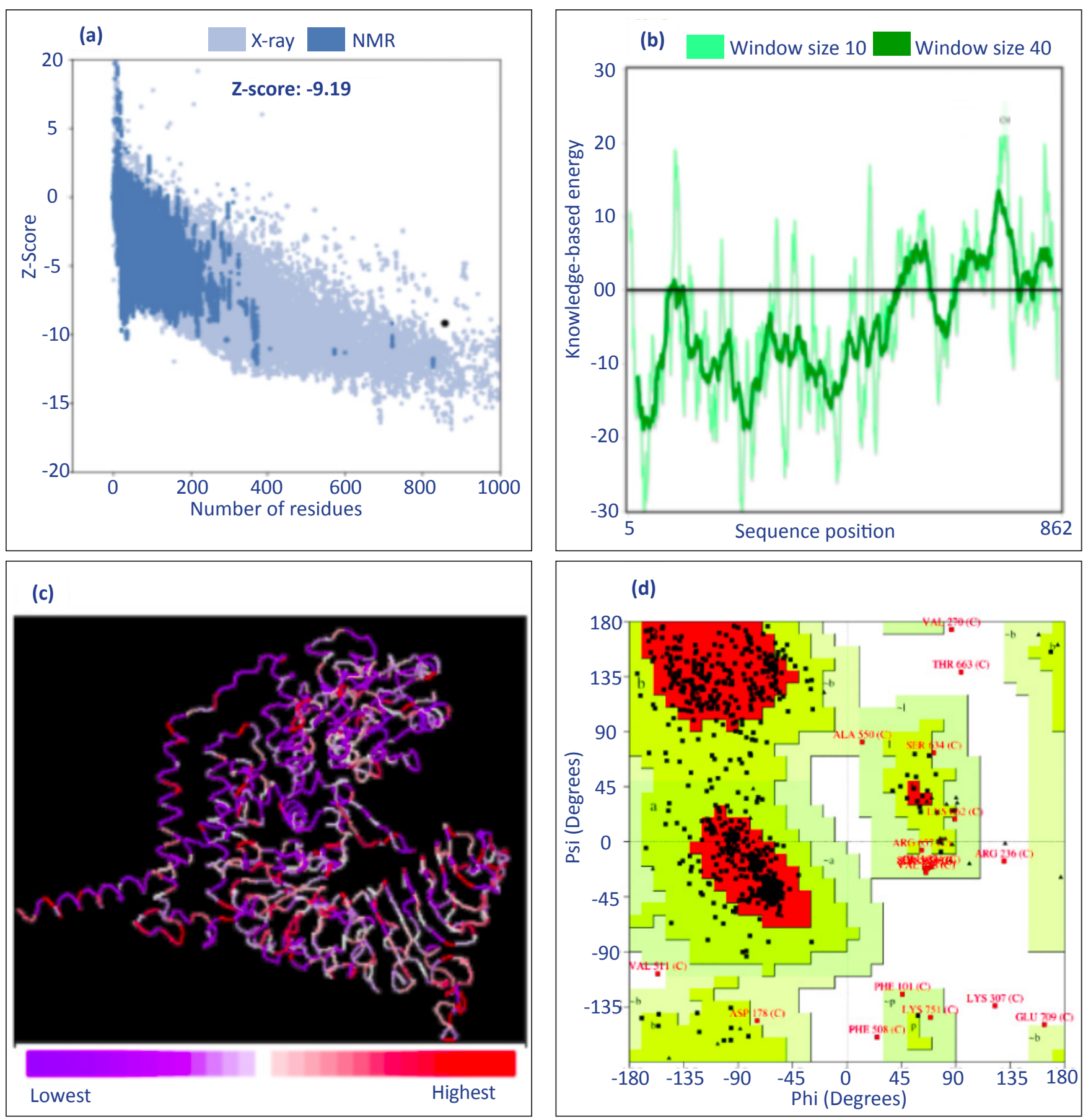

Figure 1: Evaluation of 3D-model of BjuWRR1 using ProSA tools and Ramachandran plot analysis: (a).Protein structural analysis (ProSA) of theBjuWRR1 model showing overall model quality in terms of Z-score is depicted.(b). Energy plot as a function of amino acid sequence position in the model reflecting the local quality of BjuWRR1 is shown. (c) The lowest energy rich regions of the model are shown in blue color while the highest energy rich regions of the model reflecting problematic regions are shown .(d) Stereochemical parameters of the model is represented in a Ramachandran plot.

hydrophobic amino acid residues and a number of polar amino acid residues. Oligomerization of the CC-domain is reported to play a role in the induction of defense response signaling such as hypersensitive response (Collier et al., 2011; Maekawa et al., 2011; Cesari et al., 2016). Hydrophobic and polar residues in CC-domain of BjuWRR1 as visualized in multiple sequence alignment (Figure 3) is an indicator of the potential role of these residues in the activation of downstream defense response signaling in response to white rust effector protein by promoting oligomerization between CC-domains through stabilized hydrophobic interactions and interacting with NBARC domain. 


\subsection{2. $N B-A R C$ domain}

In NLR proteins, a central NB-ARC (Nucleotide-binding adaptor shared by Apaf-1, Resistance proteins, and CED4) (van der Biezen and Jones, 1998) domain is present next to the N-terminal CC-domain. Interestingly, the NB-ARC domain is a member of signal transduction ATPases and plays an important role as a molecular switch to modulate many biological processes including immunity and apoptosis (Danot et al., 2009; Inohara et al., 2005). Analysis of our built model of BjuWRR1 revealed that it harbors a central NB-ARC domain in between $\mathrm{N}$-terminal CC-domain and C-terminal LRR domain. NB-ARC is composed of three sub-domains namely nucleotide binding domain (NBD), HD1-domain and a winged helix domain (WHD). NBD spans from Ser134 residue to Leu316 residue and is composed of a four-stranded parallel beta-sheet surrounded by a total of six alpha-helices on either side of the beta-sheet. Similarly, the HD1 sub-domain is composed of four alpha-helices spanning from Thr317 to Asp383 residues. In the BjuWRR1 model, the Winged-helix sub-domain is present in between Asn384 to Arg 529 residues and is composed of six alpha-helices and was found to harbor many disordered loops. Further inspection of the NB-ARC domain of BjuWRR1 revealed the presence of canonical conserved motifs such as Kinase-1a or P-loop, Kinase-2 motif, HD-motif. The presence of patches of charged residues in the NB-ARC domain may play a role in an intramolecular interaction with $\mathrm{N}$-terminal CCdomain to trigger a conformational change in the CC-domain for activating defense signaling response upon detection of pathogen-derived effector proteins (Figure 2 and Figure 3).

\subsubsection{LRR domain}

Leucine-rich repeat (LRR) domain of NLR proteins is highly variable and plays a vital role in the direct recognition of

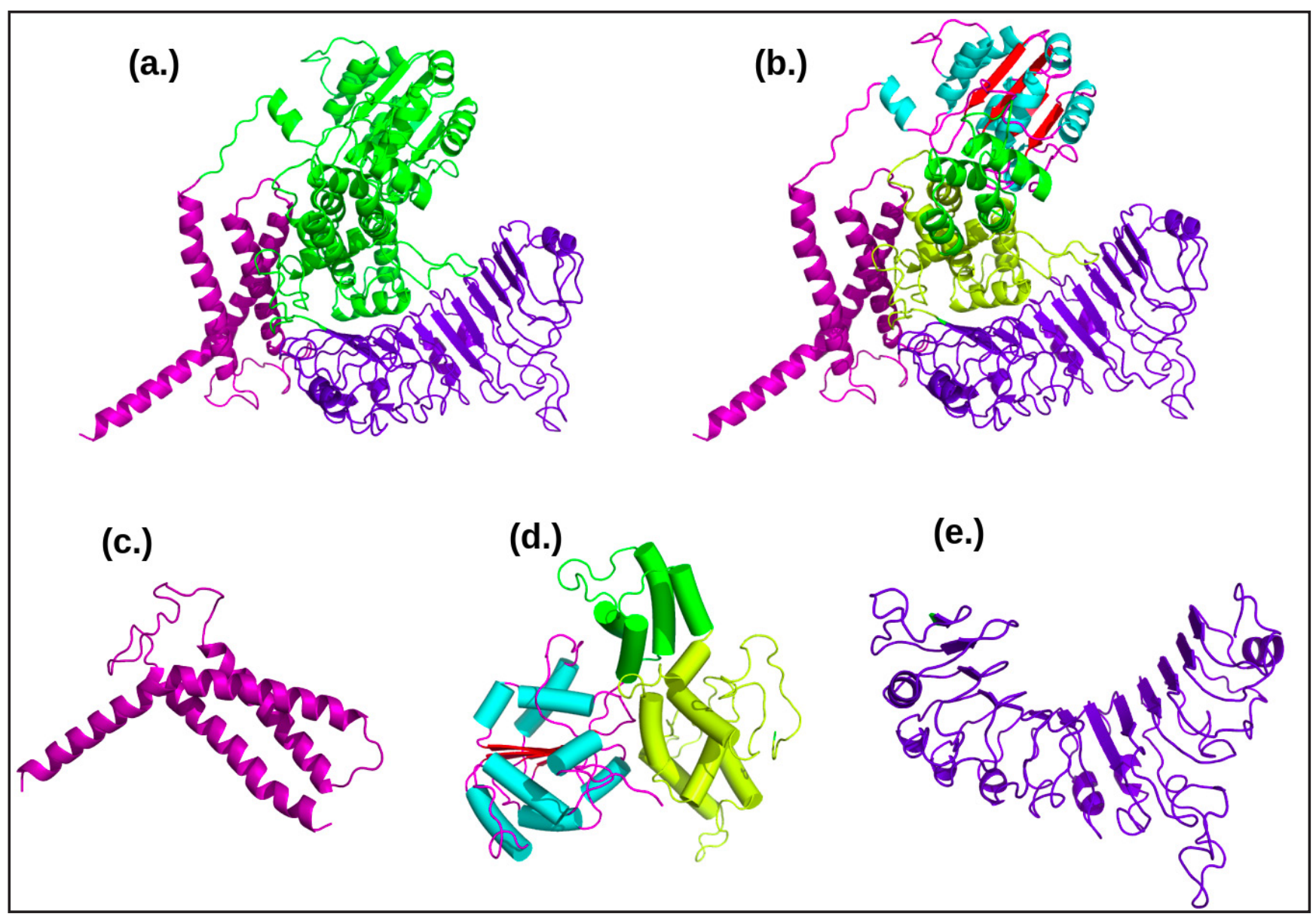

Figure 2: Molecular anatomy of CC-NBS-LRR type R-protein BjuWRR1 in Brassica juncea: The predicted three dimensional model of BjuWRR1 is shown in Figure 2a displaying different canonical domains namely a coiled-coil N-terminal domain (purple) followed by NBS domain (green) and a variable LRR domain (blue). Similarly, the entire model of BjuWRR1 is presented showing N-terminal coiled domain and C-terminal LRR domain along with different subdomains of central NBS domain namely a nucleotide binding domain (cyan-red), helical domain1(HD1) (green) and a winged helix domain (WHD) (yellow) in Figure 2b. Magnified view of N-terminal coiled coil domain in purple color and different subdomains of central NBS domain are shown respectively, in Figure $2 \mathrm{c}$ and Figure $2 \mathrm{~d}$. The typical horseshoe shaped leucine rich repeat (LRR) domain is presented in its magnified view in blue color in Figure $2 \mathrm{e}$

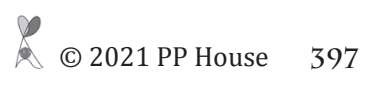




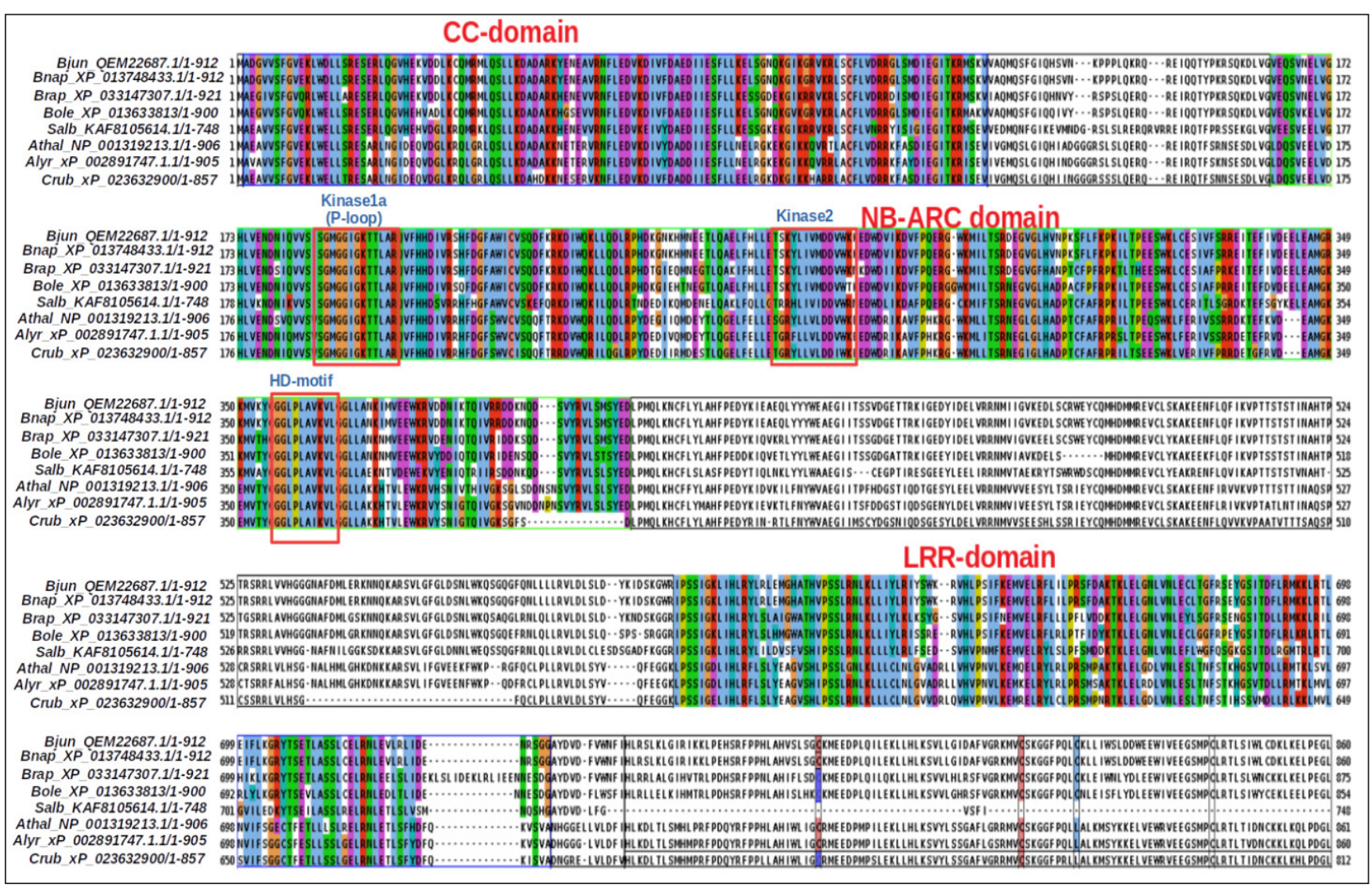

Figure 3: Multiple sequence alignment of BjuWRR1 orthologues in different plant species: Presence of three canonical conserved domains namely a coiled-coil (CC) domain at N-terminal, central NB-ARC domain and a C-terminal leucine rich repeat (LRR) domain are highlighted in red color fonts. Bjun: Brassica juncea, Brap: Brassica rapa, Bnap: Brassica napus, Bole: Brassica oleracea, Salb: Sinapsis alba, Athal: Arabidopsis thaliana, Alyr: Arabidopsis lyrata and Crub: Capsella rubella

pathogen-derived effector molecules. Further, the LRR domain also helps in keeping the NLR protein in OFF-state. Physical interaction between the LRR domain and NB-ARC is demonstrated in studies of Bs2, RPS5 and Rx in disease resistance-related cell death. (Moffett et al., 2002; Beck et al., 2012; Rairdan and Moffett, 2006). LRR is reported to play a likely role in the inhibition of autoactivation of NLR proteins such as RPS5 (Qi et al., 2012) in Arabidopsis thaliana. BjuWRR1 model was found to contain a carboxyl-terminal LRR-domain spanning from residues Val531 to Tyr862 is composed of a slender arc-shaped architecture forming a continuous betasheet along the concave surface of the arc. Each beta strand is alternate with an alpha-helix. Mostly leucine residues are found in regular intervals and hydrophobic residues are rich in LRR-domain forming a stabilized hydrophobic core which is typical to LRR protein family members (Padmanabhan et al., 2009; Kobe and Kajava, 2001). In our model, a set of conserved aspargine residues were seen in the LRR domain which may contribute to the curve-nature of LRR structures as reported by Kobe and Kajava (2001). Further, the conserved patch of lysine and other charged residues were found in the LRRdomain which may play a role in intramolecular interaction in BjuWRR1 to prevent auto activation of defense response by keeping NB-ARC domain in the off-state which in turn does not cause any conformational change in $\mathrm{N}$-terminal coiled-coil domain to initiate a downstream hypersensitive response in absence of pathogen effector molecules (Figure 2 and Figure 3).

\section{Conclusion}

A 3D-molecular model of a candidate $R$-gene encoded protein BjuWRR1 known to play a role in mediating white rust resistance in Indian mustard was developed using homology modelling. Structural analysis showed that it is composed of three canonical conserved domains (coiled-coil, NB-ARC and LRR domains). Further, presence of patches of charged residues in each domain in BjuWRR1 explains the possible role of inter-domain interaction in driving white rust resistance and can be helpful in designing of functional haplotype-based molecular markers.

\section{Acknowledgment}

Authors are highly thankful for the contribution of CKD, UN, PP, MRM, SD, PS, BK and SS. CKD conceived the idea, did the bioinformatic analysis \& wrote the manuscript. CKD, UN \& PP help in the collection of literature. MRM, SD, PS, BK and 
SS help in the proofreading of the manuscript and formating of the manuscript.

\section{References}

Anupriya, C., Shradha, N., Prasun, B., Abha, A., Pankaj, S., Abdin, M.Z., Neeraj, S., 2020. Genomic and molecular perspectives of host-pathogen interaction and resistance strategies against white rust in oilseed mustard. Current Genomics 21(3), 179-193.

Arora, H., Padmaja, K.L., Paritosh, K., Mukhi, N., Tewari, A.K., Mukhopadhyay, A., Gupta, V., Pradhan, A.K., Pental, D., 2019. BjuWRR1, a CC-NB-LRR gene identified in Brassica juncea, confers resistance to white rust caused by Albugo candida. Theoretical and Applied Genetics 132(8), 2223-2236.

Beck, M., Zhou, J., Faulkner, C., MacLean, D., Robatzek, S., 2012. Spatio-temporal cellular dynamics of the Arabidopsis flagellin receptor reveal activation statusdependent endosomal sorting. Plant Cell 24(10), 4205-4219.

Bernoux, M., Ve, T., Williams, S., Warren, C., Hatters, D., Valkov, E., Zhang, X., Ellis, J.G., Kobe, B., Dodds, P.N., 2011. Structural and functional analysis of a plant resistance protein TIR domain reveals interfaces for self-association, signaling, and autoregulation. Cell Host and Microbe 9(3), 200-211.

Borhan, M.H., Brose, E., Beynon, J.L., Holub, E.B., 2001. White rust (Albugo candida) resistance loci on three Arabidopsis chromosomes are closely linked to downy mildew (Peronospora parasitica) resistance loci. Molecular Plant Pathology 2(2), 87-95.

Borhan, M.H., Gunn, N., Cooper, A., Gulden, S., Tor, M., Rimmer, S.R., Holub, E.B., 2008. WRR4 encodes a TIRNB-LRR protein that confers broad-spectrum white rust resistance in Arabidopsis thaliana to four physiological races of Albugo candida. Molecular Plant-Microbe Interactions 21(6), 757-768.

Borhan, M.H., Holub, E.B., Beynon, J.L., Rozwadowski, K., Rimmer, S.R., 2004. The Arabidopsis TIR-NB-LRR gene RAC1 confers resistance to Albugo candida (white rust) and is dependent on EDS1 but not PAD4. Molecular Plant-Microbe Interactions 17(7), 711-719.

Cesari, S., Moore, J., Chen, C., Webb, D., Periyannan, S., Mago, R., Bernoux, M., Lagudah, E.S., Dodds, P.N., 2016. Cytosolic activation of cell death and stem rust resistance by cereal MLA-family CC-NLR proteins. Proceedings of the National Academy of Sciences 113(36), 10204-10209.

Cevik, V., Boutrot, F., Apel, W., Robert-Seilaniantz, A., Furzer, O.J., Redkar, A., Castel, B., Kover, P.X., Prince, D.C., Holub, E.B., Jones, J.D., 2019. Transgressive segregation reveals mechanisms of Arabidopsis immunity to Brassica-infecting races of white rust (Albugo candida). Proceedings of the National Academy of Sciences 116(7), 2767-2773.
Chisholm, S.T., Coaker, G., Day, B., Staskawicz, B.J., 2006. Host-microbe interactions: shaping the evolution of the plant immune response. Cell 124(4), 803-814.

Collier, S.M., Hamel, L.P., Moffett, P., 2011. Cell death mediated by the $\mathrm{N}$-terminal domains of a unique and highly conserved class of NB-LRR protein. Molecular Plant-Microbe Interactions 24(8), 918-931.

Colovos, C., Yeates, T.O., 1993. Verification of protein structures: patterns of nonbonded atomic interactions. Protein Science 2(9), 1511-1519.

Cui, H., Tsuda, K., Parker, J.E., 2015. Effector-triggered immunity: from pathogen perception to robust defense. Annual Review of Plant Biology 66, 487-511.

Danot, O., Marquenet, E., Vidal-Ingigliardi, D., Richet, E., 2009. Wheel of life, wheel of death: mechanistic insight into signaling by STAND proteins. Structure 17(2), 172-182.

Das, C.K., Bastia, D., Naik, B.S., Kabat, B., Mohanty, M.R., Mahapatra, S.S., 2018a. GGE biplot and AMMI analysis of grain yield stability and adaptability behaviour of paddy (Oryza sativa L.) genotype under different agroecological zones of Odisha. ORYZA-An International Journal on Rice 55(4), 528-542.

Das, C.K., Bastia, D., Swain, S.C., Mahapatra, S.S., 2018b. Computational analysis of genes encoding for molecular determinants of arsenic tolerance in rice (Oryza sativa L.) to engineer low arsenic content varieties. ORYZA-An International Journal on Rice 55(2), 248-259.

Das, C.K., Nayak, U., Pati, P., 2021. Elucidating the molecular anatomy of Acetyl-CoA carboxylase in Brassica rapa for evolving climate-resilient interventions to minimize carbon footprints. Sustainable Chemical Engineering 2(2), 21-39

DeLano, W.L., 2002. Pymol: An open-source molecular graphics tool.CCP4 Newsletter on protein crystallography 40(1), 82-92.

Dodds, P.N., Rathjen, J.P., 2010. Plant immunity: towards an integrated view of plant-pathogen interactions. Nature Genetics 11(8), 539-548.

Duxbury, Z., Ma, Y., Furzer, O.J., Huh, S.U., Cevik, V., Jones, J.D., Sarris, P.F., 2016. Pathogen perception by NLRs in plants and animals: Parallel worlds. Bio Essays 38(8), 769-781.

Ferreira, M.E., Williams, P.H., Osborn, T.C., 1995. Mapping of a locus controlling resistance to Albugo candida in Brassica napus using molecular markers. Phytopathology 85(2), 218-220.

Heller, A., Thines, M., 2009. Evidence for the importance of enzymatic digestion of epidermal walls during subepidermal sporulation and pustule opening in white blister rusts (Albuginaceae). Mycological research 113(67), 657-667.

Inohara, N., Chamaillard, M., McDonald, C., Nunez, G., 2005. NOD-LRR proteins: role in host-microbial interactions and inflammatory disease. Annual Review of Biochemistry 74, 355-383. 
Jeanmougin, F., Thompson, J.D., Gouy, M., Higgins, D.G., Gibson, T.J., 1998. Multiple sequence alignment with Clustal X. Trends in Biochemical Sciences 23(10), 403-405.

Jones, J., Dangl, J.L., 2006. The plant immune system. Nature 444, 323-329.

Kamoun, S., Furzer, O., Jones, J.D., Judelson, H.S., Ali, G.S., Dalio, R.J., Roy, S.G., Schena, L., Zambounis, A., Panabières, F., Cahill, D., 2015. The top 10 oomycete pathogens in molecular plant pathology. Molecular Plant Pathology 16(4), 413-434.

Kobe, B., Kajava, A.V., 2001. The leucine-rich repeat as a protein recognition motif. Current Opinion in Structural Biology 11(6), 725-732.

Kole, C., Teutonico, R., Mengistu, A., Williams, P.H., Osborn, T.C., 1996. Molecular mapping of a locus controlling resistance to Albugo candida in Brassica rapa. Phytopathology 86(4), 367-369.

Kole, C., Williams, P.H., Rimmer, S.R., Osborn, T.C., 2002. Linkage mapping of genes controlling resistance to white rust (Albugo candida) in Brassica rapa (syn. campestris) and comparative mapping to Brassica napus and Arabidopsis thaliana. Genome 45(1), 22-27.

Kumar, R., Yadav, S.S., Singh, U., Verma, H.P., 2021a. Growth, yield, quality and energetics of mustard (Brassica juncea (L.) Czern \& Coss) as influenced by weed management and sulphur fertilization under semi arid condition of Rajasthan. International Journal of Bio-resource and Stress Management 12(4), 255-263

Kumar, A., Sharma, P., Thomas, L., Agnihotri, A., Banga, S.S., 2009. Canola cultivation in India: scenario and future strategy. $16^{\text {th }}$ Australian research assembly on Brassicas. Ballarat, Victoria, 0-5.

Kumar, G., Rashid, M.M., Teli, B., Bajpai, R., Nanda, S., Yadav, S.K., 2021b. Cultivar mixture: old but impactful plant disease management strategy. International Journal of Economic Plants 8(3), 113-119

Laskowski, R.A., MacArthur, M.W., Moss, D.S., Thornton, J.M., 1993. PROCHECK: a program to check the stereochemical quality of protein structures. Journal of Applied Crystallography 26(2), 283-291.

Lee, H.A., Yeom, S.I., 2015. Plant NB-LRR proteins: tightly regulated sensors in a complex manner. Briefings in Functional Genomics 14(4), 233-242.

Links, M.G., Holub, E., Jiang, R.H., Sharpe, A.G., Hegedus, D., Beynon, E., Sillito, D., Clarke, W.E., Uzuhashi, S., Borhan, M.H., 2011. De novo sequence assembly of Albugo candida reveals a small genome relative to other biotrophic oomycetes. BMC Genomics 12(1), 1-12.

Lukasik, E., Takken, F.L., 2009. Standing strong, resistance proteins instigators of plant defense. Current Opinion in Plant Biology 12(4), 427-436.

Maekawa, T., Cheng, W., Spiridon, L.N., Toller, A., Lukasik, E., Saijo, Y., Liu, P., Shen, Q.H., Micluta, M.A., Somssich, I.E., Takken, F.L., 2011. Coiled-coil domain-dependent homodimerization of intracellular barley immune receptors defines a minimal functional module for triggering cell death. Cell Host \& Microbe 9(3), 187-199.

Meena, P.D., Verma, P.R., Saharan, G.S., Borhan, M.H., 2014. Historical perspectives of white rust caused by Albugo candida in Oilseed Brassica. Journal of Oilseed Brassica 5(Special), 1-41

Michelmore, R.W., Christopoulou, M., Caldwell, K.S., 2013. Impacts of resistance gene genetics, function and evolution on a durable future. Annual Review of Phytopathology 51, 291-319.

Moffett, P., Farnham, G., Peart, J., Baulcombe, D.C., 2002. Interaction between domains of a plant NBS-LRR protein in disease resistance-related cell death. EMBO Journal 21(17), 4511-4519.

Padmanabhan, M., Cournoyer, P., Dinesh Kumar, S.P., 2009. The leucine-rich repeat domain in plant innate immunity: a wealth of possibilities. Cellular Microbiology 11(2), 191-198.

Panjabi-Massand, P., Yadava, S.K., Sharma, P., Kaur, A., Kumar, A., Arumugam, N., Sodhi, Y.S., Mukhopadhyay, A., Gupta, V., Pradhan, A.K., Pental, D., 2010. Molecular mapping reveals two independent loci conferring resistance to Albugo candida in the east European germplasm of oilseed mustard Brassica juncea. Theoretical and Applied Genetics 121(1), 137-145.

Petit-Houdenot, Y., Fudal, I., 2017. Complex interactions between fungal avirulence genes and their corresponding plant resistance genes and consequences for disease resistance management. Frontiers in Plant Science 8, 1072.

Pettersen, E.F., Goddard, T.D., Huang, C.C., Couch, G.S., Greenblatt, D.M., Meng, E.C., Ferrin, T.E., 2004. UCSF Chimera-a visualization system for exploratory research and analysis. Journal of Computational Chemistry 25(13), 1605-1612.

Prabhu, K., Somers, D.J., Rakow, G., Gugel, R.K., 1998. Molecular markers linked to white rust resistance in mustard Brassica juncea. Theoretical and Applied Genetics 97(5), 865-870.

Pruitt, K.D., Tatusova, T., Maglott, D.R., 2005. NCBI reference sequence (RefSeq): a curated non-redundant sequence database of genomes, transcripts and proteins. Nucleic Acids Research 33(suppl_1), D501-D504.

Qi, D., Deyoung, B.J., Innes, R.W., 2012. Structure-function analysis of the coiled-coil and leucine-rich repeat domains of the RPS5 disease resistance protein. Plant Physiology 158, 1819-1832.

Rairdan, G.J., Moffett, P., 2006. Distinct domains in the ARC region of the potato resistance protein Rx mediate LRR binding and inhibition of activation. Plant Cell 18(8), 2082-2093.

Saharan, G.S., Verma, P.R., 1992. White rusts: a review of economically important species. IDRC, Ottawa, ON, CA. 
Saharan, G.S., Verma, P.R., Meena, P.D., Kumar, A., 2014. White rust of crucifers: biology, ecology and management. Springer.

Shao, Z.Q., Xue, J.Y., Wu, P., Zhang, Y.M., Wu, Y., Hang, Y.Y., Wang, B., Chen, J.Q., 2016. Large-scale analyses of angiosperm nucleotide-binding site-leucine-rich repeat genes reveal three anciently diverged classes with distinct evolutionary patterns. Plant Physiology 170(4), 2095-2109.

Sippl, M.J., 1993. Recognition of errors in three-dimensional structures of proteins. Proteins: Structure, Function, and Bioinformatics 17(4), 355-362.

Swiderski, M.R., Birker, D., Jones, J.D., 2009. The TIR domain of TIR-NB-LRR resistance proteins is a signaling domain involved in cell death induction. Molecular PlantMicrobe Interactions 22(2), 157-165.

Takken, F.L., Goverse, A., 2012. How to build a pathogen detector: structural basis of NB-LRR function. Current Opinion in Plant Biology 15(4), 375-384.

van der Biezen, E.A., Jones, J.D., 1998. The NB-ARC domain: a novel signalling motif shared by plant resistance gene products and regulators of cell death in animals. Current Biology 8(7), R226-R228.

Wang, J., Hu, M., Wang, J., Qi, J., Han, Z., Wang, G., Qi, Y., Wang, H.W., Zhou, J.M., Chai, J., 2019. Reconstitution and structure of a plant NLR resist some conferring immunity. Science 364 (6435).

Waterhouse, A., Bertoni, M., Bienert, S., Studer, G., Tauriello, G., Gumienny, R., Heer, F.T., de Beer, T.A.P., Rempfer, C., Bordoli, L., Lepore, R., 2018. SWISS-MODEL: homology modelling of protein structures and complexes. Nucleic Acids Research 46(W1), W296-W303.

Waterhouse, A.M., Procter, J.B., Martin, D.M., Clamp, M., Barton, G.J., 2009. Jalview Version 2-a multiple sequence alignment editor and analysis workbench. Bioinformatics 25(9), 1189-1191.

Wiederstein, M., Sippl, M.J., 2007. ProSA-web: interactive web service for the recognition of errors in three-dimensional structures of proteins. Nucleic Acids Research 35(suppl 2), W407-W410. 